\title{
Handwritten tifinagh character recognition using simple geometric shapes and graphs
}

\author{
Y. Ouadid, B. Elbalaoui, M. Boutaounte, M. Fakir, B. Minaoui
}

Sultan Moulay Slimane University, Faculty of Science and Techniques, Beni-Mellal, Morocco

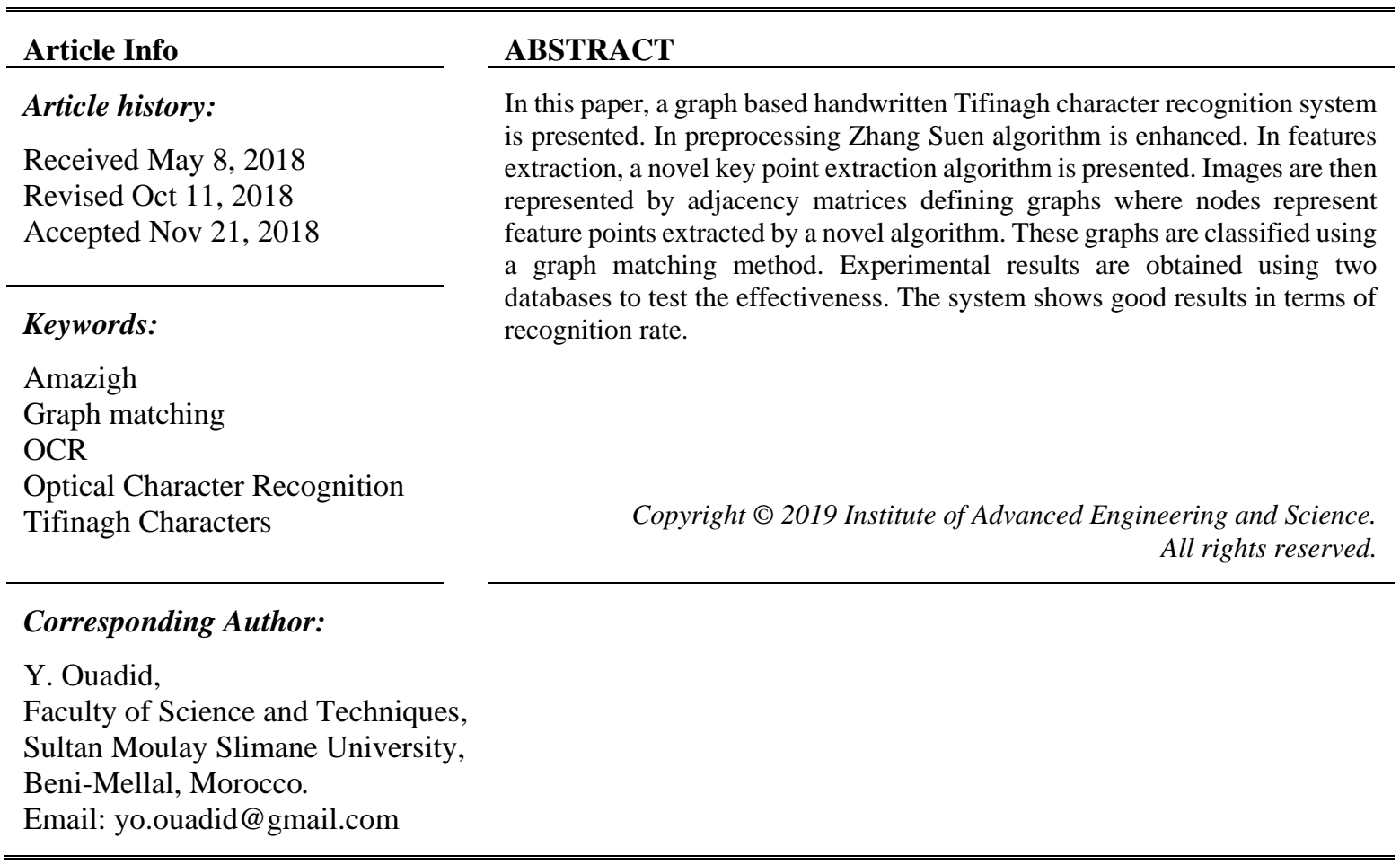

\section{INTRODUCTION}

Optical Character Recognition (OCR) is a branch of pattern recognition and artificial intelligence. It contributes enormously to human-machine communication. It is the computational process of converting images of printed or handwritten text into machine-readable text that is easily reproducible by a computer system (such as Microsoft Word). OCR is more specifically concerned with forms of information acquired from a printed paper document, such as invoices, computerized receipts, passport documents, bank statements, letters, books or any appropriate documentation. The realization of such a process is very complicated because there is an infinity of representations of writing. Indeed, each person has a unique writing style and there are many styles and fonts in printed characters. Thus, character recognition systems are adapted to the type of writing envisaged (printed, manuscript or cursive).

Thanks to recent advances in computer science, many techniques of handwriting recognition have also been improved, especially for Latin and Arabic writing [1,2]. Thus, in recent years, with the growth of the means of communication, other alphabets such as the Tifinagh alphabet of the Amazigh language have integrated in the information systems. This has led to the appearance of other types of documents where writing is not yet handled and therefore more difficult to recognize. Text recognition of such documents requires more specific processing techniques.

In this paper, the aim is to apply an OCR system on the Amazigh handwriting (Tifinagh). The Amazigh language is spoken today by a large number of populations all over the world, mainly in the North Africa. It is normally written from left to right and vertically from top to bottom. The Amazigh writing is noncursive which simplifies the segmentatiFon of characters in a text image. Figure 1 illustrates the Tifinagh characters adopted by IRCAM. 
Comparing with Roman, Arabic or Chinese, research on the recognition of Amazigh writing has not reached perfection. As far as authors know, very few attempts have been made on the recognition of Amazigh handwriting. Amrouch et al. [3] described a global approach for the recognition of handwritten Tifinagh character in which they used Hough transform as feature extraction method and Hidden Markov Models (HMM) for classification. The system produced promising result, however the discrimination of this model is not good enough due to learning step when a single image is used for every character. El Ayachi et al. [4] presented a solution of rotation and noise problems by using dynamic programming, invariant moments and Walsh transform. The system produced good results however combining multiple descriptors make the system slower. El Kessab et al. [5] combined Multi-Layer Perceptron and HMM for more discriminative system which proven to be reliable in term of recognition rate but slower in term of CPU time. Gounane et al. [6] proposed a hybrid approach by combining neural networks and b-gram. The system produced satisfactory results, however recognition rate can be improved using bigger corpus. Es-saady et al. [7] presented a system where the horizontal and vertical baseline positions are the features of the character. The system showed better results when baselines are replaced by centerline.

\begin{tabular}{|c|c|c|c|c|c|c|c|c|}
\hline ya & 。 & $\mathrm{a}$ & yah & 人 & ha & yar & $Q$ & r \\
\hline yab & $\theta$ & b & yac & ウ & $\varepsilon$ & yay & ५ & Y \\
\hline yag & X & g & yax & $x$ & $\mathrm{X}$ & yas & $\odot$ & $\mathrm{S}$ \\
\hline yagw & $7^{4}$ & $\mathrm{gW}^{\mathrm{W}}$ & yaq & Z & $q$ & yaș & O & Ș \\
\hline yad & $\Lambda$ & d & yi & $\Sigma$ & $\mathrm{i}$ & yac & 6 & c \\
\hline yaḍ & $E$ & d & yaj & $I$ & $\mathrm{j}$ & yat & $t$ & $\mathrm{t}$ \\
\hline yey & 웅 & $\mathrm{e}$ & yal & U & 1 & yat & $E$ & $\mathrm{t}$ \\
\hline yaf & ][ & f & yam & ᄃ & $\mathrm{m}$ & yaw & ப & W \\
\hline yak & R & $\mathrm{k}$ & yan & I & $\mathrm{n}$ & yay & 5 & $\mathrm{y}$ \\
\hline yak $^{w}$ & $R^{u}$ & $\mathrm{k}^{\mathrm{W}}$ & $\mathrm{yu}$ & : & $\mathrm{u}$ & yaz & 米 & $\mathrm{Z}$ \\
\hline yah & (1) & $\mathrm{h}$ & yar & 0 & $\mathrm{r}$ & yaz & 米 & Z \\
\hline
\end{tabular}

Figure 1. Tifinagh characters

Comparing with English, Arabic or Chinese, research on the recognition of Amazigh writing has not reached perfection. As far as authors know, very few attempts have been made on the recognition of Amazigh handwriting. Amrouch et al. [3] described a global approach for the recognition of handwritten Tifinagh character in which they used Hough transform as feature extraction method and Hidden Markov Models (HMM) for classification. The system produced promising result, however the discrimination of this model is not good enough due to learning step when a single image is used for every character. El Ayachi et al. [4] presented a solution of rotation and noise problems by using dynamic programming, invariant moments and Walsh transform. The system produced good results however combining multiple descriptors make the system slower. El Kessab et al. [5] combined Multi-Layer Perceptron and HMM for more discriminative system which proven to be reliable in term of recognition rate but slower in term of CPU time. Gounane et al. [6] proposed a hybrid approach by combining neural networks and b-gram. The system produced satisfactory results, however recognition rate can be improved using bigger corpus. Es-saady et al. [7] presented a system where the horizontal and vertical baseline positions are the features of the character. The system showed better results when baselines are replaced by centerline.

To summarize, all research proposed for the recognition of Amazigh handwriting used statistical approach, where features are represented as a dimensional point in the corresponding vector space, which allow the use of vector space properties to resolve classification problems.

In this work, a handwritten character recognition system based on a structural approach is proposed. In this approach the patterns are devised into several parts then represented by a graph. This graph describes the properties and spatial position of those parts including their relationship between each other.

This paper is organized as follows: in section 2, the preprocessing phase is described. Section 3 deals with the structural features extraction phase. Section 4 deals with the classification phase and finally, in the last section experimental results are discussed. 


\section{PREPROCESSING}

The purpose of preprocessing is to produce a clean version of the input image by keeping relevant information which differentiate the pattern in the feature space.

In this step, The RGB image is converted into a binary image. In fact, the well-known Otsu Method [13] is applied to the Red, Green and blue channels of the input image. Gathering those three monochrome images gives us a binary image with the maximum relevant information of the pattern. This step allowed to remove low to medium level of noise from the image.

Based on the monochrome version of the input image, the unwanted areas are removed from the image. It consists of scanning the vertical histogram horizontally and the horizontal histogram vertically to find character borders. The space between those borders and the image borders is removed.

To simplify the extraction structural feature; the normalized monochrome image is thinned using Zhang-Suen algorithm [8]. For a better thinning results, this algorithm is used as follow:

1. Apply thinning on connected components instead of applying it to the entire image. This allows us to treat the case of removed components.

2. Smooth the skeleton by eliminating pixels that do not affect the connectivity and topology of the character

Figure 2 summarize the steps of preprocessing of a handwritten Tifinagh character.

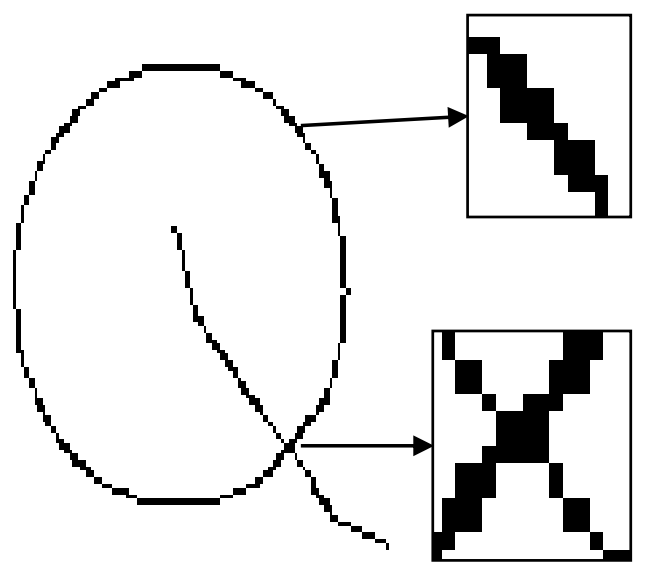

(a)

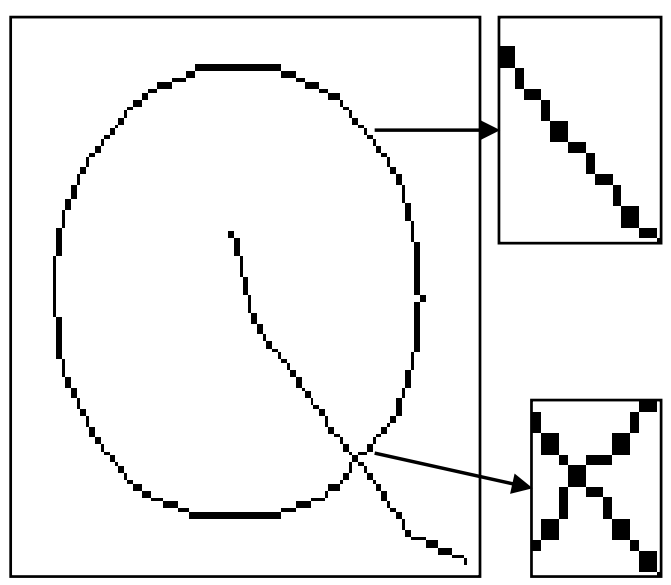

(b)

Figure 2. Thinning result example: (a) Standard thinning Algorithm, (b) Enhanced thinning algorithm

Figure 3, illustrates the execution time of the process of pre-processing of the Tifinagh handwritten characters and those of the printed characters used in previous work [11]. The preprocessing time of handwriting is obviously greater than that of the printed writing because the latter requires much more preprocessing.

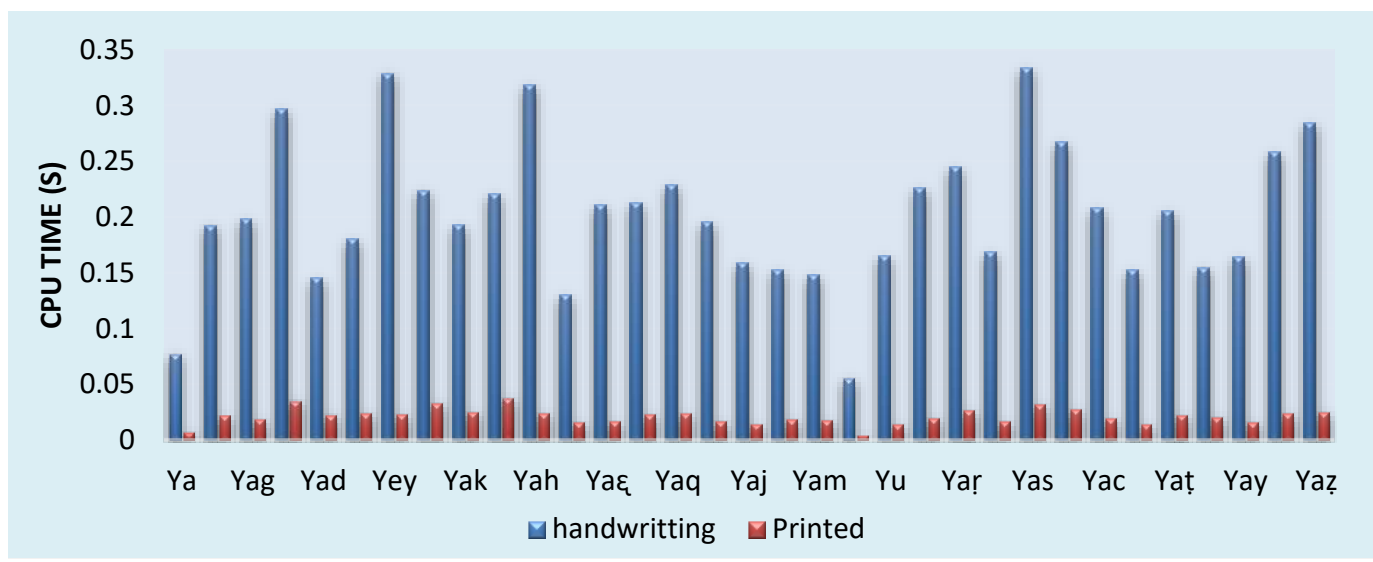

Figure 3. Pre-processing time of handwritten and printed characters 


\section{STRUCTURAL FEATURE EXTRACTION}

The structural descriptions consist of extracting the topological and geometrical properties of the character with a high tolerance to distortions and variations of style. This type of representation can also encode some knowledge about the structure of the object or can provide some knowledge about the components constituting this object.

Graph representation is one of the most adequate ways to encode the structure information of a pattern (in our case Tifinagh character). It is a formal mathematical representation of a set of objects and their relationships. Each object is called vertices. The relationships between objects are called edges. More formally, a graph $\boldsymbol{G}$ is defined as an ordered pair $\boldsymbol{G}=(\boldsymbol{V}, \boldsymbol{E})$ where $\boldsymbol{V}$ is a set of vertices and $\boldsymbol{E}$ is a set of edges that define the connectivity between a pair of vertices.

The adjacency matrix is a way to represent the graphs. It is a binary square matrix $\boldsymbol{A} \boldsymbol{M}$ where the number of vertices $|\boldsymbol{V}|$ is its size. The entry in row $\mathrm{i}$ and column $\mathrm{j}$ is nonzero if and only if the edge $(\boldsymbol{i}, \boldsymbol{j})$ is in the graph which means:

$$
\left\{\begin{array}{l}
A M(i, j)=1, \text { an edge is connecting vertice } i \text { and } j \\
A M(i, j)=0, \quad i \text { and } j \text { are not connected }
\end{array}\right.
$$

In this case, authors worked on undirected graphs whose edges direction is not important. This representation is fast and compact. However, there is a redundancy of information since the matrix is symmetric.

To represent Tifinagh characters by graphs, key points are extracted first. They will serve as delimiter for character segmentation into line segments and nodes in graph representation. In a perfect character skeleton, a foreground pixel is considered as a key point by looking to the number of foreground pixels in its neighborhood as shown in Figure 4. If the number of neighbors is one then that pixel is considered as an end point (as shown in Figure 4.a). If the number of neighbors is more than two then that pixel is an intersection point (see Figure 4.b). However, a skeleton is rarely perfect no matter what thinning algorithm is used, two or more-pixel width part are often found in the skeleton. To remedy to this issue, the number of the transitions from a background pixel to a foreground pixel is considered instead of the number of foreground pixels (see Figure 4.c). Which means that a pixel is classified as a primary key point if the number of transitions is one or more than two.

\begin{tabular}{|l|l|l|}
\hline 0 & 1 & 0 \\
\hline 0 & 1 & 0 \\
\hline 0 & 0 & 0 \\
\hline
\end{tabular}

(a)

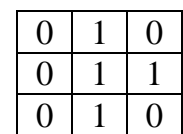

(b)

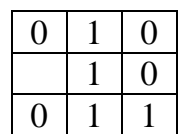

(c)

Figure 4. Example of pixel neighborhood, (a): end point, (b): intersection point, (c): none key point pixel since the number of transactions from white pixel to black pixel is two

End and intersection points are not enough to divide the character skeleton into several line segments. Secondary key points are needed between segments that are curvier then straight. To do so, an algorithm is proposed which is similar to the algorithm authors proposed in [14] that allows to divide a curvy segment into several line segments. It is based on the fact that the smallest distance between two points (in our case those two points are the end of segments extracted based on end and intersection points) is the straight line connecting them.

The algorithm starts by extracting segments between primary key points (end and intersection points). Every segment is classified into a straight line or curvy line. This is done using a threshold which is the length of segment $\boldsymbol{D}$ divided by the Euclidian distance $\boldsymbol{d}$ its end. The value of the threshold, calculated empirically, is 0.2 . The example for "yah" character key points extraction using authors proposed algorithm shown in Figure 5.

$$
\left\{\begin{array}{l}
\frac{d}{D} \geq 0.2 \text { the segment is an arc } \\
\frac{d}{D}<0.2 \text { the segment is a ligne }
\end{array}\right.
$$




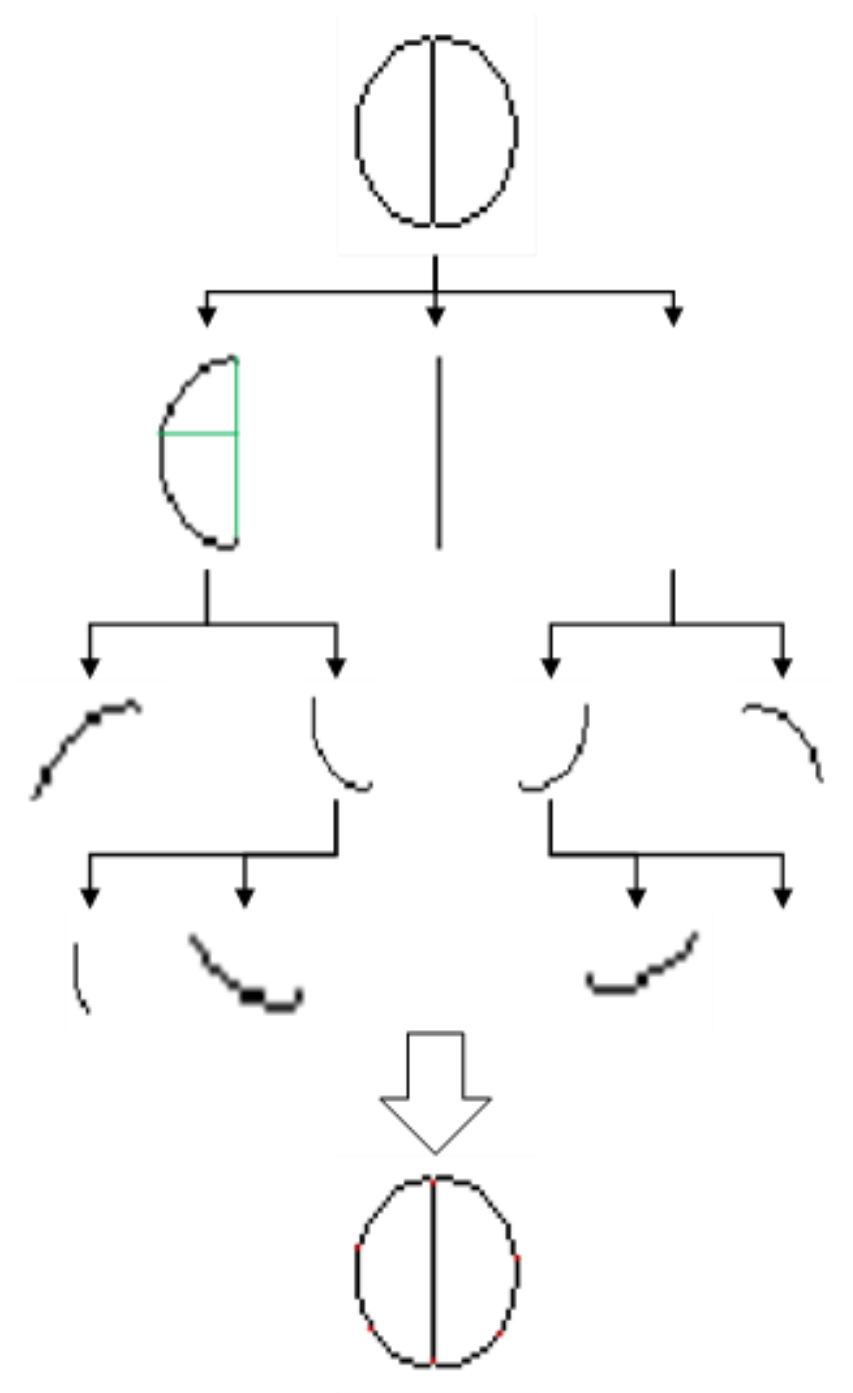

Figure 5. "yah" character key points extraction using authors proposed algorithm

When an arc is detected, the orthogonal distance between the segment elements and the straight line is calculated. The element that has the maximum perpendicular distance is designated as an inflection point. Every time a point is added to a segment, two new segments replace the former segment in the segment list. The algorithm used for the extraction of the key points is as follows:

1. Extract primary key points.

2. Extract segments based on these points.

3. For every segments, checks if it is an arc.

4. For every arc, make the point that have the biggest orthogonal distance as a key point.

5. Divide arc segment into two segments where the new key points become an end of the new segments.

6. Update the list of segments.

7. Repeat step 3 to 6 until no arc segment is detected.

To evaluate the performance of the proposed algorithm, for each Tifinagh handwritten character, the number of key points extracted by this algorithm is compared to that extracted by the well-known Harris algorithm [15] and the optimal number of points (calculated empirically) necessary for the graphical representation; and on the other hand, the execution times of these algorithms. This comparison, illustrated in Figure 6 and 7, shows that our algorithm is more efficient than Harris in terms of the speed and the extraction of the key points. 


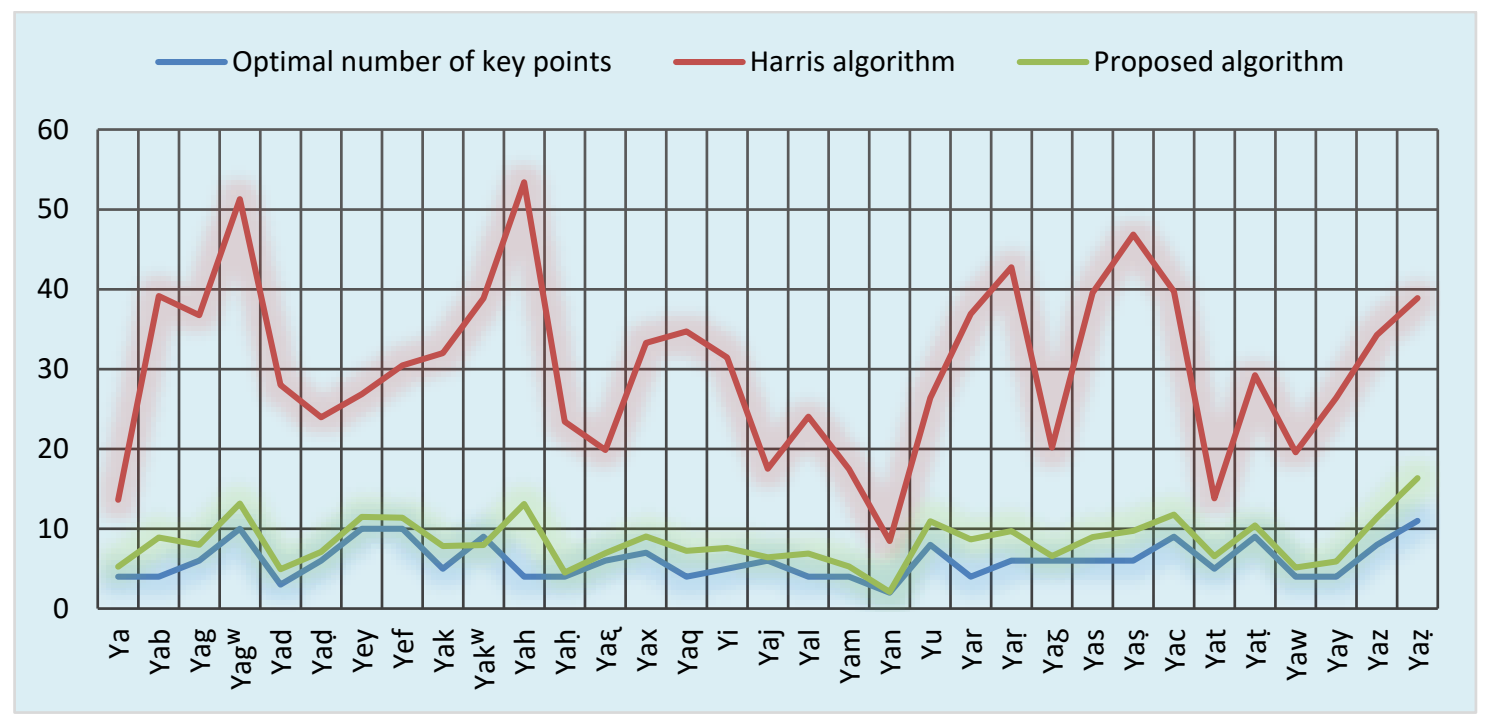

Figure 6. Comparison of the number of points obtained by the Harris method, the proposed algorithm and the optimal number of points

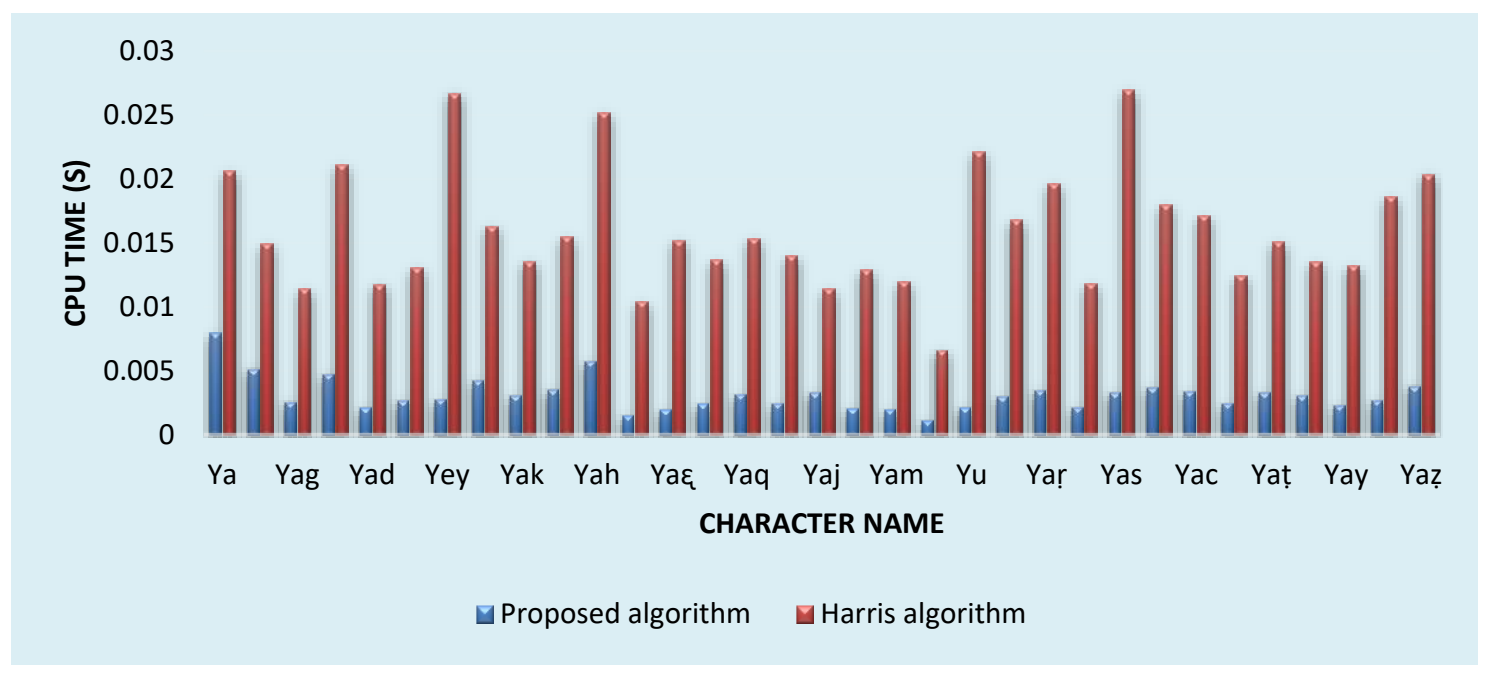

Figure 7. Execution time of the Harris algorithm and the proposed algorithm, case of handwritten Tifinagh characters

Now that the character skeleton is divided into several line segments and key points, a graphical representation can be applied where the nodes represent the key points and edges represent segments connecting these points in pairs. The graph construction algorithm is proceeded as follows:

1. Let $A M$ be an $n \times n$ adjacency matrix where $\mathrm{n}$ is the number of key points.

2. For every segment, four information are extracted: first key point, second key point, segment orientation $O$, segment length $L$.

3. Based on this information, the adjacency matrix is constructed as follow:

$\operatorname{AM}(i, j)=\omega$, key point $i$ is connected to key point $j$

$\{\operatorname{AM}(i, j)=0$, else

Where,

$\omega=2 \times \mathrm{L}+0$ 
This representation is faster than in the case of the use of key points extracted by Harris. This is illustrated in Figure 8, which gives the execution time of the algorithm of the graphical representation for each character.

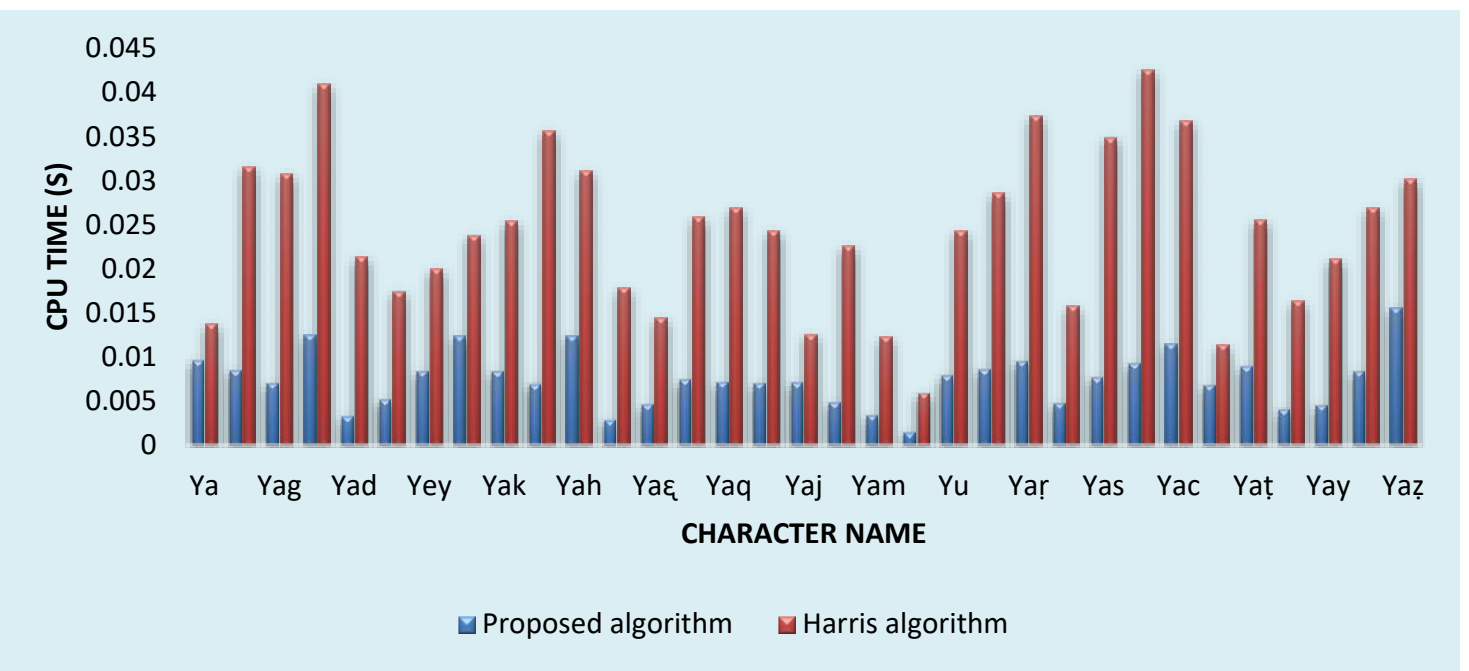

Figure 8. Execution time of the graph representation algorithm characters per key points extracted by the algorithm and Harris algorithm

\section{CLASSIFICATION}

The used spectral method [9] aims at finding a coherent agreement between two graphs. This is done by calculating Eigen values and Eigen vectors of the graph product (or Similarity matrix) of the two graphs. the biggest Eigen value of the graph product designs the degree of similarity between the two graphs. Readers are invited to check the original paper that is cited for more details.

\section{EXPERIMENTAL RESULTS}

To evaluate the overall performance of the proposed system, authors used two database sets of handwritten Tifinagh characters. The first database [10] is composed of 1376 images; 43 images for each character. It was created with the help of 30 people and other 13 to take in consideration inclination issues. The second database [12], is composed of 25740 images; 780 images for each character. It was created with the help of 60 person with deferent ages. During tests, 80\% (1100 images for the first database and 20592 images for the second database) of these databases are used as reference images and 20\% (276 images for the first database and 5148 images for the second database) are used as test images. These experiments are done using a laptop with $2.6 \mathrm{GHz}$ speed i7 dual core processor, $8 \mathrm{~GB}$ RAM and MATLAB software.

The recognition rate results obtained are illustrated in Table 1 . A recognition rate of $94 \%$ is obtained on the first database and $85 \%$ for the second database. The decreased recognition rate from the first to the second database is due to the increasing of badly written characters, the increasing of noise level or the loss of important information in the acquisition phase.

Table 1. comparison of recognition and error rate of the proposed system with other systems

\begin{tabular}{lllll}
\hline & & First database [10] & Second database [12] & Local Database \\
\hline Authors proposed & Recognition rate (\%) & 94 & 85 & - \\
system & Error rate (\%) & $\mathbf{6}$ & $\mathbf{1 5}$ & - \\
Amrouch et al. [3] & Recognition rate (\%) & - & - & 90.4 \\
& Error rate (\%) & - & - & $\mathbf{9 . 6}$ \\
\multirow{2}{*}{ El Kessab et al. [5] } & Recognition rate (\%) & - & - & 92.3 \\
& Error rate (\%) & - & - & $\mathbf{7 . 7}$ \\
Es-saady et al. [7] & Recognition rate (\%) & - & 94.96 & - \\
& Error rate (\%) & - & $\mathbf{5 . 0 4}$ & - \\
\hline
\end{tabular}




\section{CONCLUSION}

In this paper, recently, proposed Optical Character Recognition (OCR) system is applied to handwritten Tifinagh characters. The main contribution of this paper is the application of a novel key point extraction technique and graph matching on handwritten Tifinagh characters. This technique showed great results in term of pattern description and speed compared to the well-known Harris method. Experimentation on two different databases proved the good performance of the system. However, many enhancements are required, especially in the classification process. A better classification technique will be proposed in the future work.

\section{REFERENCES}

[1] Mansi S, Jethava G B. A Literature Review On Hand Written character Recognition. Indian Streams Research Journal. $2013 ; 3(2)$.

[2] Lorigo M L, Govindaraju V. Offline Arabic handwriting recognition: a survey. IEEE Transactions on Pattern Analysis and Machine Intelligence; 2006.

[3] Amrouch M, Rachidi A, El Yassa M, Mammass D. Handwritten Amazigh Character Recognition Based On Hidden Markov Models. December 2010; ICGST-GVIP Journal, 10(5).

[4] El Ayachi R, Moro K, Fakir M, Bouikhalene B. On The Recognition of Tifinaghe Scripts. Journal of Theoretical and Applied Information Technology. 2010.

[5] El Kessab B, Daoui C, Moro K, Bouikhalene B, Fakir M. Recognition of Handwritten Tifinagh Characters Using a Multilayer Neural Networks and Hidden Markov Model. Global Journal of Computer Science and Technology. September 2011; 11(15).

[6] Gounane S, Fakir M, Bouikhalen B. Handwritten Tifinagh Text Recognition Using Fuzzy K-NN and Bi-gram Language Model. IJACSA Special Issue on Selected Papers from Third international symposium on Automatic Amazigh processing (SITACAM’13). Beni Mellal, 2013.

[7] Es-Saady Y, Amrouch M, Rachidi A, El Yassa M, Mammass D. Handwritten Tifinagh Recognition Using Baselines Detection Features. International Journal of Scientific \& Engineering Research. 2014; 5(4).

[8] Zhang T Y, Suen C Y. A Fast-Parallel Algorithm for Thinning Digital Patterns. Communications of the ACM. 1984; 27(3).

[9] Leordeanu M, Hebert M. A spectral technique for correspondence problems using pairwise constraints. International Conference of Computer Vision. 2005.

[10] Bencharef O, Chihab Y, Mousaid N, Oujaoura N. Data set for Tifinagh handwriting character recognition. Data in Brief. 2015; 4:11-13.

[11] Ouadid Y, Fakir M, Minaoui B. Tifinagh Printed Character Recognition through Structural Feature Extraction. International Journal of Computer Vision and Image Processing (IJCVIP). 2016; 6(2): 42-53.

[12] Es Saady Y, Rachidi A, El Yassa M, Mammass D. AMHCD: A Database for Amazigh Handwritten Character Recognition Research. International Journal of Computer Applications. August 2011; 27(4): 44-48.

[13] Otsu N. A Threshold Selection Method from Grey-level Histograms. IEEE Transactions on systems, Man and Cybernetics. 1979; SMC-9: pp. 62-66.

[14] Boutaounte M, Ouadid Y. Tifinagh Characters Recognition Using Simple Geometric Shapes. Indonesian Journal of Electrical Engineering and Computer Science. 2016 Jul 1;3(1):235-9.

[15] Harris C, Stephens M. A combined corner and edge detector. Proceedings of the Fourth Alvey Vision Conference, University of Sheffield Printing Unit, Manchester. 1988; pp.147-151. 